\title{
Towards more humanized real time decision support systems
}

\author{
F.G. Filip \\ Research Institute for Informatics \\ 8-10, Averescu Avenue 71316 Bucharest, Romania \\ Phone: +40-1-2223778 Fax: +40-1-3128539 \\ E-mail: FILIPF@ROEARN.ICI.RO
}

\begin{abstract}
This paper aims at reviewing several results concerning the development of practical Decision Support Systems (DSS) for real time production control in manufacturing. Several characteristic features of DSS that are highly demanded and/or accepted by human factors and mimic the human problem solving are identified. The perspective includes technology, business and human factors. A practical DSS illustrates the approach.
\end{abstract}

\section{Keywords}

Decision support systems, intelligent control, human factors, levels of automation, process industry

\section{INTRODUCTION}

In order to make industrial enterprises survive under sharp economic competition, engineers have for many years been striving at building large integrated computer based management and control systems. Various terms and concepts such as 'plant wide control '- PWC (in continuous process industries), 'computer integrated manufacturing '- CIM (in discrete part manufacturing) illustrate a holistic approach based on computing and communication facilities thereby to automate/support, and integrate various automatable or less automatable engineering, production and management activities (Williams, 1990). Recently, besides communications, extensive use of AI techniques (Kusiak, 1992) appears to be an important technology driving force. This latest development is mainly tributary to a ' knowledge based production' trend that might sometimes be too difficult to reflect in case of SME which cannot afford hiring highly qualified and highly paid personnel.

Other recent concepts such as Computer Integrated Enterprise - CIE or Computer Integrated Business - CIB (Raulefs, 1994), are examples of a less technology-driven, but apparently more business-oriented perspective, and illustrate an even broader approach of automation and computer based integration concept. 
Moreover recent evolutions such as globalisation of markets, better informed clients, knowledge based products, integration of SMEs into global production, new enterprise paradigms (Norman, 1994), and business process reengineering - BPR (Hammer and Champy, 1993), to integrate people, technologies, production and business, are supported by existing information technologies. In turn, these developments exert significant impact on information systems (IS) development (Granado, 1993).

In addition to technology and business efficiency factors, a third element namely the human factor, should be taken into account in modern IS. A human centred approach would also include human centred goals such as job satisfaction, transparency, knowledge enrichment, understandability, etc. (Johannsen, 1994).

A preliminary analysis of various interacting internal influence factors (information technologies and control methodologies) and of external factors, was reported (Filip, 1994).

A series of articles (Filip et al, 1985; Filip,1988;Filip,1993) has been produced on concepts and methods devised or adopted in evolving a family of practical decision support systems for manufacturing (DSSAM) addressing production control in continuous process industries (refineries, chemical plants, pulp and paper mills) as well as in discrete part manufacturing (DPM) workshops.

This paper focuses on human factors (HF), with particular emphasis on DSSfM. The paper is organised as follows. A general discussion of HF is made in Section 2. Section 3 reviews several relevant aspects of real time (RT) DSSfM. A practical RT DSSeM evolution is shown in Section 4.

\section{HUMAN FACTORS}

Johannsen (1994) remarks that 'in addition to CIM, it seems to be necessary to also consider human integrated manufacturing (HIM) during system development. In a human centered design approach the question how to integrate the humans on several or all levels of the plant has to be dealt with during early systems development phases'.

In general, one can identify three main groups of questions about the interaction and integration of the human and the machine (the IS): 1 . how does the computer serve the human (process/plant manager) perform his tasks better?; 2. what is the impact of the man - machine system on the operational performance of the managed process/plant? ; 3 . how the human status and working conditions are affected by the presence of computer?

Most of the early IS have not been used as expected because of being unreliable, intolerant (requiring an absolutely correct stream of directives to carry out their functions), impersonal (the dialogue was not consistent with user's previous experience) and not self sufficient (the help of the computer professional has been frequently needed ) (James, 1980). While most of those early critical issues have been solved so far by technology developments and intensive ('continuous' and 'accelerated') training of users, rendering the information tool less impersonal is still an open problem, at least in process supervision and production control.

To answer the second question, a separate analysis of the performances of the information tool, as made by Hill et al (1993), starting from the management system model, though interesting , might prove irrelevant if not completed with a clear definition of user classes (roles) and of their tasks. A recent study in the world of SME (Halsall et al, 1994) showed that 'many smaller companies lacked a clear understanding of many of the elements of sound production management practice'. Hence a 'normative use' of IS in an attempt at changing working styles, is desirable. In addition, in the 
industrial process automation context, the process efficiency is less important than safety considerations. As Johannsen (1994) remarked, the tendency to continue automating in a technology-driven manner led to 'deskilling the human operators, and thus to boredom under normal operational conditions as well as to human errors in emergency situations'. Even though with Johannsen physical process control counts, it is human errors that may bear on physical safety and also economically at the higher decision levels such as production control and plant management.

This latter observation set the stage for (and partially answered) the third question. Many years ago, Briefs (1981) stated rather dramatically that the computerisation of human work seemed to imply a 'major threat against the human creativity and conscious development, since there is a tendency to polarise humans into two categories. The first group includes computer professionals who manifest and develop their skills and creativity in designing ever more sophisticated tools. The second group would include communities of computer users who fast and easily perform their jobs without getting deep insights into their comfortable production means'.

The previously presented conclusion (Filip, 1989) is that it is necessary to develop IS that are not only precise, cheap and easy to use, but also stimulating for users as to acquire new skills, adopt new working styles and deploy their talents and creativity', is still valid. It is difficult to propose a methodology for attaining these rather general goals. An imperfect and incomplete refinement of the above goals made from a pure human centred perspective, could now cover several subgoals/attributes of IS such as wide (not 'Procustian') range of services offered ('physiology' dimension), evolutive development and possible learning facilities ('ontogeny' dimension), and transparency on system structure and explanation features (" anatomy' dimension). A particular subclass (component) of IS, namely DSS, is discussed in the sequel, trying to outline its specificities.

\section{REAL TIME DSS FOR MANUFACTURING}

Most of the traditional developments in the DSS domain have addressed systems not involving any real time control (Charturverdi et al, 1993). In the sequel, real time decisions for control applications in manufacturing will be considered. Bosman (1987) stated that control problems could be looked upon as a 'natural extension' and as a 'distinct element' of planning decision making processes (DMP).

Real time (RT) DMP for control applications in manufacturing are characterized by several particular aspects: 1 . they involve continuous monitoring of the dynamic environment; 2 . they are short time horizon oriented and are carried out on a repetitive basis; 3 . they normally occur under time pressure; 4. long-term effects are difficult to predict (Charturverdi et al, 1993). It is quite unlikely that an 'econological' (Bahl and Hunt, 1984) approach, involving optimisation, be technically possible for 'pure' RT DMP. Satisfactory approaches, that reduce the search space at the expense of the decision quality, or fully automated DM systems (corresponding to the 10th degree of automation in Sheridan's (1992) classification), if taken separately, cannot be accepted either, but for some exceptions.

At the same time, one can notice that 'pure' RT DMP can be come across in 'crisis' situations only. For example, if a process unit must be shut down, due to an unexpected event, the production schedule of the entire plant might turn obsolete. The right decision will be to take the most appropriate compensation measures to 'manage the crisis' over the time period needed to recompute a new schedule or update the current one. In this case, a satisfycing decision may be appropriate. If the crisis situation has been previously met with and successfully surpassed, an almost automated 
solution based on past decisions stored in the IS can be accepted and validated by the human operator. On the other hand, the minimisation of the probability of occurrences of crisis situations should be considered as one of the inputs (expressed as a set of constraints or/and objectives) in the scheduling problem. For example in a pulp and paper mill, a unit plant (UP) stop may cause drain the downstream tank (T) and overflow the upstream tank and so, shut/slow down the unit plants that are fed or feed those tanks respectively. Subsequent UP startings up normally imply dynamic regimes, that determine variations of product quality. To prevent such situations, the schedule (the sequence of UP production rates) should be set so that stock levels in Ts compensate to as large extent as possible for UP stops or significant slowing down.

To sum up those ideas, one can add other specific desirable features to the particular subclass of IS used in manufacturing control. An effective real time DSS for manufacturing (RT DSSfM) should support decisions on the preparation of 'good' and 'cautious' schedules as well as 'ad hoc', pure RT decisions to solve crisis situations. An example comes to illustrate this approach.

\section{DISPATCHER - A DSS SERIES}

DISPATCHER is a series of DSSs, developed over a fifteen-year time period, to solve various DMPs in the milieu of continuous 'pure material' process industries. The system initially addressed the short-term production scheduling problem. Then it evolved in both function set supported and new technologies used in order to satisfy users' various requirements (see.Figure l). New supported functions such as tank sizing, maintenance planning and even order acceptance and planning of raw materials or/and utility purchasing allow a certain degree of integration of functions within the enterprise.

\subsection{Application Area}

A typical plant in process industry consists of several (tens) of unit plants interconnected via tanks or directly through pipelines. The planning decision problem lies in choosing (over a number of time intervals) the sequence of operation regimes (configuration problem-CP) and production rates of UP (flow scheduling problem-FSP) so that the stock levels should be in the vicinity of some safe and economical values and product deliveries should be made in due time, given the initial stocks and the forecasts on material inputs. In order entry applications, or in crisis situations, orders are not treated as external 'fixed'/given consumers (C) but as free decision variables. In a similar way, raw material and industrial utility flows are viewed as external resources $(\mathbf{R})$ in ordinary scheduling problems, but as constrained decision variables in crisis situations and purchasing decisions. More details about the problem (including mathematical model and algorithm) together with numerical examples can be found in (Filip, 1990, 1993).

\subsection{Evolution}

Initially, DISPATCHER was a pure interactive optimisation program (running on PDP-DEC 11 machines) to quickly solve large scale FSP represented as linear, quadratic , discrete time, constrained, optimal tracking models. The model took stock levels for state variables $(x(k))$ and production rates for control variables $(\boldsymbol{u}(\mathrm{k}))$. The sequences of desired safe states, recommended 


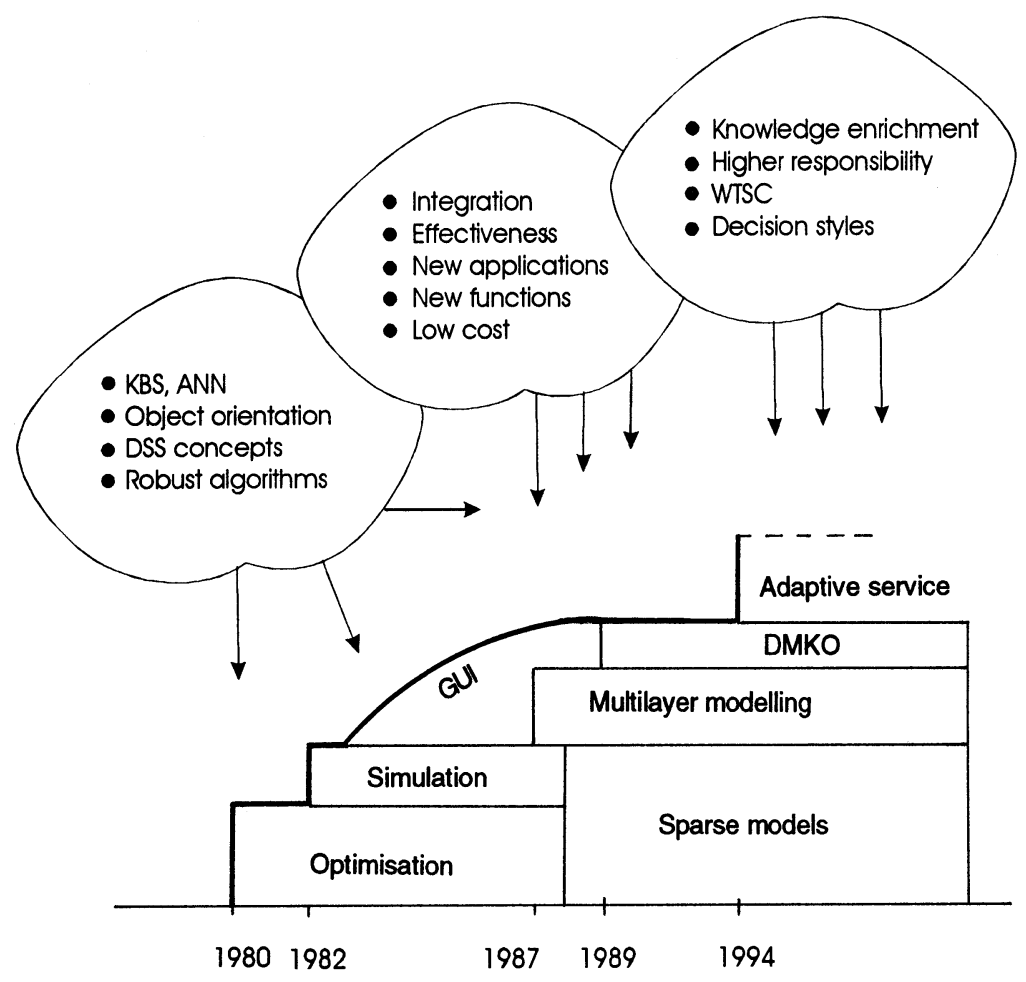

Figure 1 Technology, business and human influence factors and DISPATCHER development layers

controls, and forecast disturbances (material inputs and product deliveries) $(x d(k+1), u d(t) w(k)$ were set by the decision maker over the planning horizon with $\mathrm{KF}$ time intervals together with the values of interconnection matrices $(\boldsymbol{A}(k), \boldsymbol{B}(k))$, permitted variation limits $(\boldsymbol{x l}, \boldsymbol{x u}$ and $\boldsymbol{u l}, \boldsymbol{u} \boldsymbol{u})$ and penalties on deviations from the expected values as diagonal matrices $(Q(k), R(k))$.

$\boldsymbol{x}(k+1)=A(k) x(k)+B(k) \mathbf{u}(k)+w(k)) ; k=1, \mathrm{KF}$

$x(k+1) \in[x l(k+1), x u(k+1)] ; u(k) \in[u l(k), u u(k)] ; k=1, \mathrm{KF}$

$\min _{x, u}\left[\sum_{k}\|x(k+1)-x d(k+1)\|_{Q(k)}^{2}+\|u(k)-u d(k)\|_{R(k)}^{2}\right]$ 
This has been solved by a fast, robust, hierarchical optimisation algorithm (Filip et al, 1985). Large scale problems with up to 80 constrained states, 60 constrained controls and 12 time intervals could be solved for practical applications within a few minutes. Simple simulation facilities to support 'What if...?' analysis ( mainly concerning the alterations of computer optimal solutions or of entirely human made schedules) and negotiation processes were offered to users adopting 'implicit favourite' or ' bounded rationality' decision styles. User-friendly graphic user interface (GUI) including a specialised editor, was developed.

Many practical applications show the problem parameters (limits, desired values) which, although variable in time, keep nevertheless constant over some time segments. This and the users' demand for a reduction of the amount of data to input into the system, as well as the necessity of easily deducing one problem specification from the previous one (to evaluate several alternatives), contributed to devising a new storage method and the corresponding set of solving algorithms for sparse models with relatively constant parameters (Filip, 1988). At the same time, other roles such as application system builders (facilitators) and developers of alternative algorithms (the 'toolsmiths') had to be considered along with production planners. Consequently, a multilevel modelling scheme of the problem, with three layers (external/representational, mathematical/conceptual and internal/performance) and translation mechanisms were brought in, being inspired by parallel developments in database technology, and anticipating some recent issues related with knowledge engineering (Filip, 1993). The standard version of the system is presented in detail by (Filip, 1990).

\subsection{Towards intelligent DSS}

Numerous other practical implementations of the standard version helped draw interesting conclusions. First, the system has been considered by most users as being flexible enough to support a wide range of applications and, in some cases, its utilisation migrated from the originally intended one. It has been used in crisis situations (mainly due to significant deviation from the schedule, to equipment failures or other emergencies) as well as in normal operation or in training applications. However, though the system is somehow transparent, and the users have sound domain ("what type?') knowledge (DK), they have behaved in a 'wise' or even 'lazy'(Rasmunsen, 1983) manner, mainly trying to keep their mental load under an average 'willing to spend capacity' (WTSC). This can be explained by the initial lack of tool ('how'-type) knowledge (TK) as well as by insufficient work motivation.

To fight the lack of TK and to stimulate users' creativity and quest for new skills, a declarative model of an 'ambitious' and knowledgeable operator (DMKO) was proposed (Filip, 1993). DMKO supports 1. model building for various situations (to solve $\mathrm{CP}$ ); 2. problem feasibility testing to propose corrective measures ( for example limit relaxations or transformation of fixed/known perturbations into free variables, etc.); 3 . automatically building the internal model from the external description, choosing the appropriate solving algorithm ; 4. experimenting the problem model, for example by producing a series of alternatives through modifying various parameters (mainly weights) in answer to qualitative assessment of simulated solutions by the user, followed by due explanations. To handle the complexity and diversity of the technologies used, object orientation has been adopted. 
Efforts are being made to introduce new intelligence into the system, especially for evaluating users' behaviour so that DMKO (originally meant for supporting a certain 'role') could dynamically adapt to specific needs of particular 'actors', in an attempt at rendering the system less ' impersonal'.

Of course, there are other reported results combining traditional numeric methods with KBS to build 'hybrid' (Nof and Grant,1991) or 'tandem' DSSfM (Kusiak, 1992) even in process industries (Dourado-Correia, 1992). Apparently such systems are primarily meant for making numerical computation easier, including heuristics so that the space search for optimisation/simulation algorithms is adapted/reduced. It should be noted that the author's approach is mainly human factor centred and aims at increasing system acceptance rather than improving its computational performances. However it is felt that this solution, though technically interesting, together with a more intensive training, cannot always overpass the motivation problem. This problem can apparently be handled by organisational measures within an BPR effort.

\section{CONCLUSIONS}

Problem solving (PS) process can be considered from different perspectives. Human PS is a combination of calculation, reasoning and adaptive learning. Automatic PS is a combination of such elements as numeric, symbolic, and neural computing (Kerckhoffs, 1994).

Intelligent DSS include learning facilities, perform inferences and select appropriate heuristics, rules and models (Sen, 1993). These technologies mimic the activity of human brain as a whole, and represent an important step forward in the movement towards humanizing the modern information systems, in order to make them more compatible with human decision maker. In addition to those remarkable technology developments, a human centred perspective was intended and pleaded for in this paper.

\section{6}

\section{REFERENCES}

Bahl, H.C. and Hunt, R.G. (1984) Decision making theory and DSS design. Data Base, 15(4), . 10 14.

Briefs, U. (1981) Re-thinking industrial work: computer effects on technical white collar workers. Computers in Industry, 2(1), 76-89.

Bosman, A. (1987) Relations between specific DSS, Decision Support Systems, 3, 213-224.

Chaturverdi, A.R., Hutchinson, G.K. and Nazareth, D.L. (1993) Supporting complex real-time decision making through machine learning. Decision Support Systems, 10, 213-233.

Dourado-Correia, A. (1992) Optimal Scheduling and Energy Management in Industrial Complexes: Some New Results and Proposals, in Proceedings on Computer Integrated Mamufacturing in Process and Mamufacturing Industries, IFAC Conference ,Espoo.

Filip.F.G. (1988) Operative Decision Making in the Process Industry, in Preprints, IMACS'88 12th World Congress, Paris.

Filip, F.G. (1989) Creativity and decision support systems. Studies and Researches in Computers and Informatics, 1(1), 41-49.

Filip, F.G. (1990) Decision support systems in process coordination, part 2: technology issues. Studies and Researches in Computers (new series), 1(4), 27-45. 
Filip, F.G. (1993) An Object Oriented Multilayer Model for Scheduling, in Proceedings, European Simulation Symposium-ESS'93 (eds. A. Verbraeck and E.J.H. Kerckhoffs), Delf .

Filip, F.G. (1994) Evolutions in systems analysis, modelling, and simulation in computer based industrial automation. Systems Analysis,Modelling and Simulation, 15, 135-149.

Filip, F.G., Donciulescu, D.A., Gaspar, R., Orasanu, L. and Muratcea, M. (1985) Multilevel optimisation algorithms in computer aided production control in the process industries. Computers in Industry, 6(1), 47-57.

Granado, J. (1994) Business Technology and Information Technology, in Annex VI to Documentation the Working Group on TBP, V 2.0, Brussels.

Halsall, D.N., Muhlemann, A.P. and Price, D.H.R. (1994) A review of production planning and scheduling in smaller manufacturing companies in the UK. Production Planning and Control, 3(5), 485-493.

Hammer, M. and Champy, J. (1993) Reengineering the firm. Harper Collins Books, New York.

Hill, D.T., Koelling, C.P. and Kurstedt, H.A. (1993) Developing a set of indicators for measuring information-oriented performance. Computers in Industrial Engineering, 24(3), 379-390.

James, K.M. (1980) The user interface. The Computer Journal, 23(1), 25-28.

Johannsen, G. (1994) Integrated Systems Engineering: The Challenging Cross-discipline, in Preprints on Integrated Systems Engineering, IFAC Conference, Pergamon Press.

Kerckhoffs, E.J.H. (1992) Parallel Processing in Model-based Problem Solving, in Computational Systems Analysis (ed. A. Sydow) Elsevier, Amsterdam.

Kusiak, A. (ed.) (1992) Intelligent Design and Manufacturing. John Wiley \& Sons, New York.

Nof, S.Y. and Grant, F.H. (1991) Adaptive/predictive scheduling: a review and general framework. Production Planning and Control, 2(4), 298-312.

Norman, D. (1994) Emerging Models of the Enterprise, in Technology for the Business Process, CEC European IT Conference, 91-92.

Rasmunsen, J. (1983) Skills, roles, and knowledge; signal signs and symbols and other distinctions in human performance models. IEEE Transactions on Systems, Man and Cybernetics SMC 13, 257-266.

Raulefs, P. (1994) The Virtual Factory, in IFIP Transactions (eds. K. Brunnstein and E. Raubold)

A52, 13th World Computer Congress'94, , Elsevier Science, Amsterdam.

Sen, M. (1993) Machine learning methods for intelligent decision support. Decision Support Systems, 10, 79-83.

Sheridan, T. (1992) Telerobotics, automation and human supervisory control. MIT Press.

Williams, T.J. (1989) A reference model for Computer integrated mamufacturing: a description from the viewpoint of industrial automation. ISA, Triangle Park.

F.G.Filip was born in Bucharest (1947). He took the MSc. degree and Ph.D degree from the 'Politehnica' University of Bucharest in 1970 and 1982, respectively. In 1991 he was elected as a corresponding member of the Romanian Academy. He is director of the Research Institute for Informatics in Bucharest. His main scientific interests are in large scale systems, optimisation and control, DSS, CIME, man-machine systems, technologies for the business processes. He published over 120 technical papers. 